\title{
The Extent and Use of Artificial Intelligence to Achieve the Big Four Agenda in Kenya
}

\author{
Mvurya Mgala \\ Institute of Computing and Informatics, Technical University of Mombasa, P.O. Box 90420 - 80100, \\ Mombasa, Kenya \\ Corresponding author: mmgala@tum.ac.ke
}

\begin{abstract}
A rtificial Intelligence (AI) is the human-like intelligence given to systems and demonstrated by machines in doing tasks associated with human intelligence. AI systems can therefore, think like humans, act like humans, think rationally or act rationally. AI systems are not only more powerful and more useful than ordinary computers, but they also solve complex emerging human problems. These systems can be used in areas such as medicine, to conduct guided surgery; transportation, for autonomous vehicle control; face recognition; speech recognition; decision making in agriculture, manufacturing and housing. The present study is a review of the AI technology trend. A search was conducted on literature, technology magazines, and other internet sources. A discussion is presented on how AI systems have been widely applied in the developed world and the extent these systems are being embraced in other countries like China to spar development. Thereafter, this paper discusses the extent to which AI has been applied in the specific areas that relate to the Kenyan Big Four Agenda. The contribution of this paper is a framework that the Kenya Government can adapt in the application $\mathrm{AI}^{\prime}$ s world transforming technologies to achieve its development agenda. We discuss the challenges that hinder full application of the AI technology and present the way forward.
\end{abstract}

Key Words: Artificial intelligence, Data mining, Machine learning, Robotics, Big Four Agenda, Kenya

\section{Introduction}

Artificial Intelligence (AI) is what is given to a system so that it behaves, thinks, or acts like it has human-level intelligence. AI has often been seen in science fiction as robots with human-like characteristics. AI entails all manner of prediction systems, such as predicting pregnancy disorders among women (Maylawati et al., 2017) . AI is the new paradigm in the phases of industrial revolution (Stearns, 2018). This revolution which started with mechanisation, followed by extensive use of electrical energy, to universal use of digitization lately uses AI technologies for more efficient manufacturing systems (Lasi et al., 2014).

Developed countries such as the US have invested in AI research centred in robots and pattern recognition. For example, Microsoft has invested in real-time robots and innovative technologies for image processing (Arora et al., n.d.). Amazon has used AI to develop autonomous robots to be used in their delivery system (Shavarani et al., 2018). Facebook has come up with an AI technology for facial recognition (Mann \& Smith, 2017). Further, robot cars have been developed at Stanford
University. Similarly, cleaning and 4-foot walking robots have been developed at Massachusetts Institute of Technology (Bledt et al., 2018). The Japanese are determined to utilise state-of-the-art artificial intelligence and ICT innovation to solve various human problems as they seek to rapidly advance (Tanaka \& Sakata, 2016).

AI has proved capable of supporting sustainable development because of the technologies that have been integrated in vast areas of decision making such as, in social media, search engines, agriculture, medicine, transport, manufacturing, and in judiciary. In Judiciary, for example, AI has been used to analyse documents and data during court proceedings so as to make speedy judgments. Research shows efforts are being made towards AI systems being able to make better legal decisions in the court rooms ( $\mathrm{Lu}$ et al., 2018). AI has indeed found use in the real world. According to $\mathrm{Ng}$, (2017), AI is set to have the same impact like electricity that transformed the world in transport, manufacturing, agriculture and health. AI is one of the most rapidly growing technologies which is also increasingly affecting our lives. It is seen as the future, offering great opportunities for all human 
beings. The methods AI uses include data mining, machine learning, robotics, natural language processing, expert systems, and computer vision.

This paper therefore, addresses the following questions: 1) what examples of AI usage exist in the 4 specific areas of the Kenyan Big Four Agenda? and 2) what model could Kenya adapt to benefit from the AI technologies in meeting her Big Four Agenda? The Big Four Agenda for Kenya and the world's Sustainable Development Goals (SDGs) have to be achieved to address challenges faced by the human kind. Therefore, the contribution of this paper is the proposed model that Kenya could embrace to leverage on AI technology to ensure rapid development.

\section{AI in Agriculture}

In agriculture, data mining as a method of $\mathrm{AI}$ has been used for decision making. A number of data mining techniques including artificial neural networks, Bayesian networks and support vector machines have been used (Kodeeshwari \& Ilakkiya, n.d.). Data mining was specifically used to understand the relationships of various climate and other factors on crop production using a large dataset of agricultural data in India (Gandhi \& Armstrong, 2016). It was therefore, possible to predict crop yield by integrating seasonal and spatial factors by using GIS technologies.

Machine learning method has also been used for enhancing the efficiency of agricultural activities. Techniques such as support vector machines and neural networks have been used as classifiers and k-means have been used for clustering (Behmann et al., 2015) These techniques have been applied to create models that have been used to create applications for early detection of plant diseases based on spectral features and weed detection based on shape descriptors with supervised or unsupervised learning methods (Behmann et al., 2015).

Further, robots have also found use in agriculture. Robots carry out complex series of actions automatically, especially one programmable by a computer. Specifically, development in robotics and sensing technologies have been applied in agribusiness models (Shamshiri et al., 2018). Agricultural systems that employ robots have made crop production more efficient and sustainable. Sensing technologies have been used by livestock farmers to assist in the management of animal health and welfare (Neethirajan et al., 2017). Soil quality has been automatically monitored and maintained. Pests and diseases have been eliminated without resorting to indiscriminate use of agrochemicals and in Netherlands, robots have been used to pick fruits and vegetables growing in greenhouses (Masuzawa et al., 2017). In the United Kingdom, robots have been used to harvest strawberry faster than human beings (King, 2017).

\section{AI in Manufacturing}

Artificial Intelligence has emerged as a game changer in manufacturing. It has grown from science fiction to a technology for improved industry manufacturing. $\mathrm{AI}$ is at the centre of the next generation of industrial systems known as Industry 4.0 (Lee et al., 2018). A study conducted in China, proposed a framework of a data-driven optimization of production. The framework allows managers to keep track of activities in a factory without their presence. It provides abstraction tools to enable decision making in addition to automatic allocation of resources, thus enabling the factory to operate almost autonomously (Arvind \& Bourne, 2016).

Machine learning as an AI method has successfully been applied in manufacturing in such areas as optimization, control and troubleshooting (Wuest et al., 2016). Similarly, data mining has been applied by exploiting vast amounts of data collected from product and process design, assembly, materials planning, quality control, scheduling, maintenance, and fault detection. The useful knowledge extracted from these processes has been of benefit in improving the industrial processes (Choudhary et al., 2008).

Efforts have been made towards intelligent manufacturing and new models have been proposed in China. One such model has a life cycle of manufacturing which uses autonomous sensing with interconnection, collaboration, learning, analysis, cognition and decision making to enhance production and improved services to users ( $\mathrm{Li}$ et al., 2017). The proposed model ensures integration of applications to form an ecosystem of intelligent manufacturing (Fig. 1). 


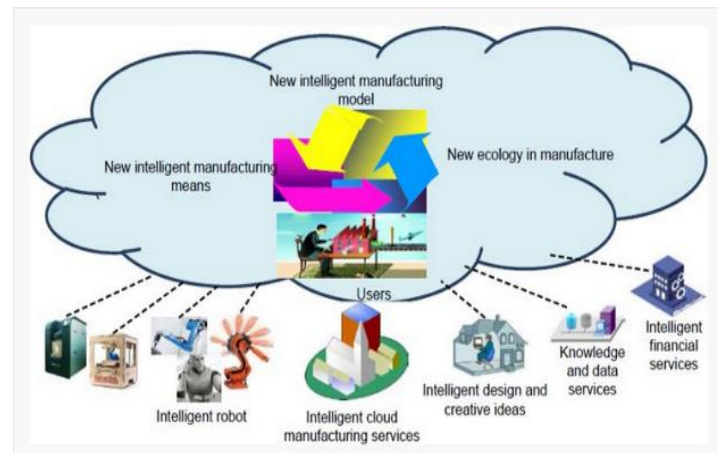

Figure 1. A proposed model for the ecosystem intelligent manufacturing ( $\mathrm{Li}$ et al., 2017)

\section{AI for Affordable Housing}

Artificial Intelligence has been applied to benefit the housing industry. AI algorithms such as Artificial Neural Networks (ANNs) have been used in the evaluation of market values for house price properties. In a research conducted in Italy, accurate results were obtained in the evaluation of house property valuation (Morano et al., 2015). Similarly, prizes of old houses have also been predicted to determine their value some years later. Such a prediction was conducted using an AI algorithm known to support vector machine where good results with an acceptable flexibility were obtained (Fu, 2018).

A related study on housing was conducted in Malaysia to predict the demand and a suitable site for affordable housing. The study developed an integrated framework of ANNs and GIS. The results of the study showed the possibility of a maximum take-up rate for the affordable houses built. Additionally, the supply and demand mismatch was eliminated (Maimun et al., 2018; Fig. 2).

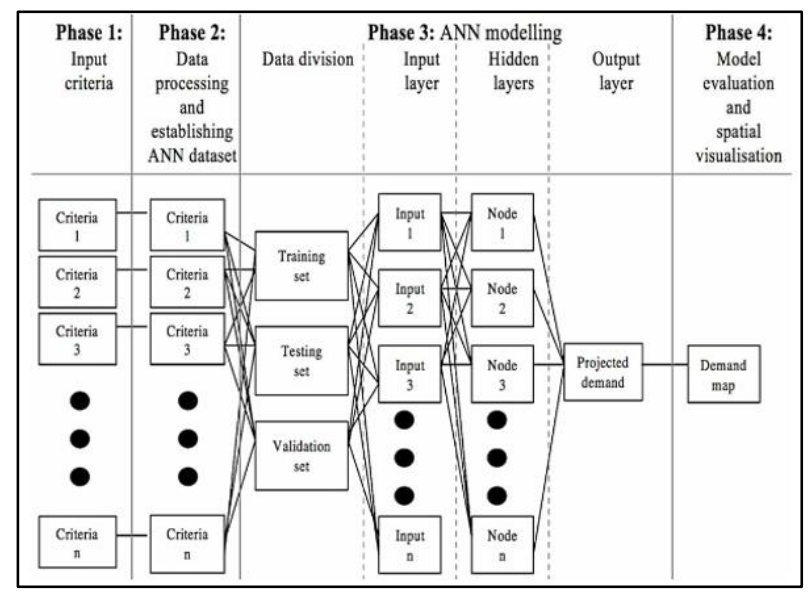

Figure 2. A demand prediction framework for affordable housing (Maimun et al., 2018)

The application of AI in achieving the Kenya Government agenda of providing affordable housing would ensure maximum utilization of resources as clearly demonstrated in the aforementioned studies.

\section{AI for Universal Health Coverage}

Artificial Intelligence has also been utilized in the medical industry. AI, using data mining has been applied in heart disease prediction. In a study conducted in India, Artificial Neural Networks was used and compared with Naive Bayes and Decision Tree. ANNs achieved the best prediction for the heart disease (Chadha et al., 2016).

AI models have been developed which have the ability to better predict patients with heart disease that surpasses medical experts. This gives further evidence that $\mathrm{AI}$ is able to improve healthcare in the world. In another study, a large dataset of over 80,000 patient records was collected and used to build the model (AI beats doctors at predicting heart disease deaths, n.d.). In a similar study, data was gathered from a wearable heart rate sensor and the model built was able to predict heart disease related conditions such as diabetes and high blood pressure with over $80 \%$ accuracy, comparable better than tests made by human experts (Ballinger et al., 2018).

Machine learning has been used to care for individual patients in medical practice. In another study, patients suffering from cancer were assessed using computers. This reduced the diagnosis time and the need for human expert, beside improving patient treatment (Darcy et al., 2016). Similarly, machine learning techniques have been used to model the progression and treatment of cancerous conditions. A study conducted early diagnosis of cancer where patients were classified accurately as high or low risk by applying machine learning methods and using techniques, such as ANNs, Bayesian Networks, Support Vector Machines and Decision Trees (Kourou et al., 2015). Such diseases as heart and cancer are killing many people in Kenya. Ensuring universal availability of techniques for diagnosis and treatment using AI techniques would assist in meeting the government agenda of achieving universal health care. 


\section{Extent of AI technologies' use in Africa}

It is evident that there is scanty use of AI technologies in Africa. Not much has been achieved using data mining methods in agriculture, education, or manufacturing. This may be attributed to lack of infrastructure for collecting data. However, attempts have been made in West Africa to improve the economic situation and food security for the people in this area by classifying rural agricultural soils (Heil et al., 2017).

Machine learning has also been scarcely used in areas such as education in Africa with rear examples such as (Mgala \& Mbogho, 2015) who predicted students in need of high intervention early enough in Kenya. Another machine learning project was conducted to predict poverty in 5 African countries - Nigeria, Tanzania, Uganda, Malawi, and Rwanda. ANNs was trained to identify image features that can explain up to $75 \%$ of the variation in local-level economic outcomes (Jean et al., 2016). In Egypt, there was effort to utilise AI technologies as seen in the book of Advanced Machine Learning Technologies including machine learning in Arabic text recognition, machine learning in watermarking authentication, feature selection, and rough sets for heart valve disease diagnosis (Hassanien et al., 2014).

Some effort to improve the use of AI technologies in East Africa is seen through the establishment of UN Pulse Lab in Uganda. The Lab explores the use of artificial intelligence in a number of areas such as the analysis of public radio discussion, use of social media to inform governance and to support response to disease outbreaks (Hagg et al., 2018). Similar efforts have been made in South Africa in establishing a national research network that carries research in various areas of AI. They have established 5 centres in universities: the University of Cape Town, KwaZulu-Natal, North West University, University of Pretoria and Stellenbosch. Research indicate that AI can improve the economy of Countries and accelerate the rate of profitability by applying automation provided by AI to a significant level (Nabi, 2019). However, it has turned out that most African Countries are not ready to take advantage of even a fraction of the potential of AI. There are indeed challenges that hinder the exploitation of AI technologies in Africa.

\section{Challenges of AI Adoption in Africa}

Africa has so far used AI technologies to a very limited extent. Although, large volumes of data are generated, there is lack of planning, investment and policy-making required to reap the full benefit of this game changing technologies. This is because there is lack of infrastructure to collect large volumes of data generated. Government infrastructure is lacking to collect for example student data in education or government databases not available for health records in hospitals and clinics. Thus, volumes of data are present but scattered and therefore not usable. In short there is lack of high quality data that could be fed into the systems.

The main challenges facing Africa can be grouped into two: scarcity of quality data and scarcity of talent. Talent is necessary because AI systems require customization, AI systems cannot be downloaded and applied to a generic problem. The scarcity of experts has limited the number of researchers in Africa that could conduct research and use AI technologies.

Limited funding from the African governments is another challenge. African researchers depend to a large extend on funding from external sources. This further hinders relevant research in AI technologies that is applicable locally. Notably, a heavy financial investment is inevitable in data mining projects since they require large volumes of data that are collected over a long period of time in addition to repeated training when developing the technologies. This calls for African governments to invest in research.

Another challenge is the lack of policy in data use. Most foreign companies that collect useful data from African citizens, such as communication companies and banks, do not release data to researchers, because it is stored in their databases. Government policies on data availability from such institutions would greatly help to create a level ground on AI research in Africa. These aforementioned challenges have formed a basis for the creation of a model that Kenya could adopt to realise her Big Four Agenda for development.

\section{The Proposed AI Model for Kenya}

If Africa as a whole, and Kenya in particular were to achieve their development agenda, the utilization of AI is inevitable. AI has been leveraged 
in developed countries to benefit in areas such as transport, manufacturing, agriculture and health. As discussed earlier, developed countries have been benefiting from AI technologies because they have heavily invested in AI research. Therefore, there is need for Kenya to adopt a model that could improve her capacity in embracing AI technologies.

This paper proposes a model that could motivate Kenya to leverage on AI technologies in order to achieve her Big Four Development Agenda (Figure $3)$. This model shows how data policy if created could facilitate the creation of government-owned databases. These databases should be availed to universities where AI research centres have been established. The AI centres will nurture talented AI researchers so that there is a pool of domain experts in the 4 areas of focus that form the Big Four Agenda.

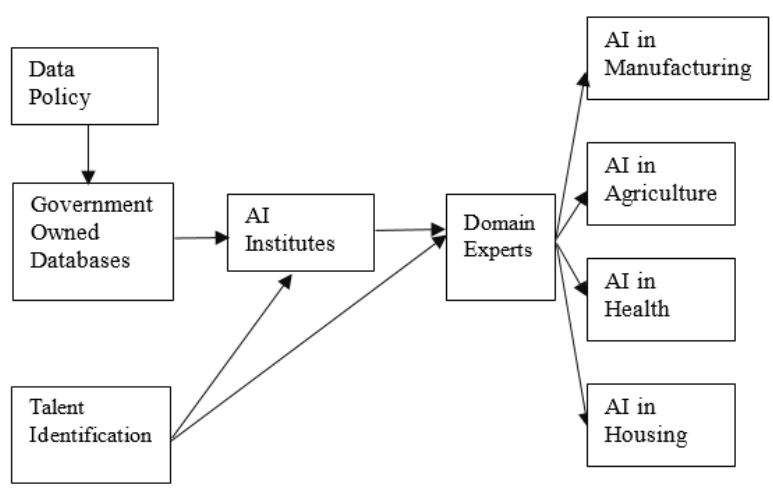

Figure 3. Proposed AI model for Kenya's Big Four Agenda

\section{Conclusion and Recommendations}

This paper has discussed the application of AI technologies as applied in the developed world to achieve development, especially in those areas in line with the Kenyan Big Four Agenda. It is clear from the above examples that AI has been used in agriculture and could be leveraged to enhance agricultural outputs and help Kenya to meet its food security agenda. Similarly, diseases such as heart and cancer that are killing many people in Kenya could be overcome by ensuring universal availability of techniques for diagnosis and treatment using AI techniques. Such effort would assist in meeting the government agenda of achieving universal health care.

AI has proved its potential in transforming the world in manufacturing, agriculture, health and housing just like electricity did 100 years ago. Africa seems to have lagged behind in leveraging this technology. The main challenges being scarcity of quality data and talent. These challenges are real in Kenya even as the country puts forward the Big Four Agenda. This paper has proposed an AI model that if adopted could motivate and make this country benefit from AI technologies so as to achieve her desired Big Four Agenda.

Clearly, from the extent in which AI technologies have made an impact in the developed countries, the Kenya Government urgently needs to invest in the process of AI technologies adoption. The many benefits that result from the use of these technologies should motivate the government to set aside funds to improve data collection infrastructure. They should finance universities to embark on research related to these technologies as a way of developing local skills, knowledge and expertise to take full advantage of these technologies. There is also need for the government to create data policies on internal data storage and data protection. The policies should go a long way to enforce availability of data for research from multinational companies such as the communication industries and banks. In future, an actual survey on the extent of AI technologies being used in Kenya and its impact should be conducted to establish their benefits.

\section{Acknowledgement}

I would like to thank Technical University of Mombasa for providing the conducive environment and motivation to conduct the research and final publication of this study.

\section{References}

AI beats doctors at predicting heart disease deaths. (n.d.). Crick. Retrieved 13 September 2018, from https://www.crick.ac.uk/news/201809-04_ai-beats-doctors-at-predicting-heartdisease-deaths

Arora, R., Sharma, J., Mali, U., Sharma, A. \& Raina, P. (n.d.). Microsoft Cognitive Services. 4.

Arvind, A. \& Bourne, D. (2016). Architecture for Industry 4.0 based Manufacturing Systems. Degree of Master of Science in Robotics. School of Computer Science Carnegie Mellon University. 
Ballinger, B., Hsieh, J., Singh, A., Sohoni, N., Wang, J., Tison, G.H., Marcus, G.M., Sanchez, J. M., Maguire, C., Olgin, J. E. \& Pletcher, M. J. (2018, April 26). DeepHeart: SemiSupervised Sequence Learning for Cardiovascular Risk Prediction. ThirtySecond AAAI Conference on Artificial Intelligence. Thirty-Second AAAI Conference on Artificial Intelligence. https://www.aaai.org/ocs/index.php/AA AI/AAAI18/paper/view/16967

Behmann, J., Mahlein, A.-K., Rumpf, T., Römer, C. \& Plümer, L. (2015). A review of advanced machine learning methods for the detection of biotic stress in precision crop protection. Precision Agriculture, 16(3), 239-260. https://doi.org/10.1007/s11119-014-9372-7

Bledt, G., Powell, M.J., Katz, B., Carlo, J.D., Wensing, P.M. \& Kim, S. (2018). MIT Cheetah 3: Design and Control of a Robust, Dynamic Quadruped Robot. 2018 IEEE/RSJ International Conference on Intelligent Robots and Systems (IROS), 2245-2252. https://doi.org/10.1109/IROS.2018.859388 5

Chadha, R., Mayank, S., Vardhan, A. \& Pradhan, T. (2016). Application of Data Mining Techniques on Heart Disease Prediction: A Survey. In N. R. Shetty, N. H. Prasad, \& N. Nalini (Eds.), Emerging Research in Computing, Information, Communication and Applications (pp. 413-426). Springer India.

Choudhary, A.K., Harding, J.A. \& Tiwari, M.K. (2008). Data mining in manufacturing: A review based on the kind of knowledge. Journal of Intelligent Manufacturing, 20(5), 501. https://doi.org/10.1007/s10845-0080145-x

Darcy, A.M., Louie, A. K. \& Roberts, L.W. (2016). Machine Learning and the Profession of Medicine. JAMA, 315(6), 551-552. https://doi.org/10.1001/jama.2015.18421

$\mathrm{Fu}, \quad$ T. (2018, June). Forecasting Second-hand Housing Price using Artificial Intelligence and Machine Learning Techniques. 2018 8th International Conference on Mechatronics, Computer and Education Informationization (MCEI 2018). https://doi.org/10.2991/mcei-18.2018.54

Hagg, E., Dahinten, V.S. \& Currie, L.M. (2018). The emerging use of social media for health- related purposes in low and middle-income countries: A scoping review. International Journal of Medical Informatics, 115, 92-105. https://doi.org/10.1016/j.ijmedinf.2018.04. 010

Hassanien, A.E., Tolba, M. \& Azar, A.T. (2014). Advanced Machine Learning Technologies and Applications: Second International Conference, AMLTA 2014, Cairo, Egypt, November 28-30, 2014. Proceedings. Springer.

Heil, J., Häring, V., Marschner, B. \& Stumpe, B. (2017, November). Classification of West African (peri)-urban and rural agricultural soils based on mid-infrared diffuse reflectance spectroscopy (DRIFT) and multivariate statistics and data mining. Jahrestagung der DBG 2017: Horizonte des Bodens, Göttingen. http:/ / eprints.dbges.de/1550/

Jean, N., Burke, M., Xie, M., Davis, W.M., Lobell, D. B. \& Ermon, S. (2016). Combining satellite imagery and machine learning to predict poverty. Science, 353(6301), 790-794. https://doi.org/10.1126/science.aaf7894

King, A. (2017, April 26). Technology: The Future of Agriculture [Comments and Opinion]. Nature. https://doi.org/10.1038/544S21a

Kodeeshwari, R.S. \& Ilakkiya, K.T. (n.d.). Different Types of Data Mining Techniques Used in Agriculture-A Survey. International Journal of Advanced Engineering Research and Science, 4(6).

Kourou, K., Exarchos, T.P., Exarchos, K.P., Karamouzis, M.V. \& Fotiadis, D.I. (2015). Machine learning applications in cancer prognosis and prediction. Computational and Structural Biotechnology Journal, 13, 817.

https://doi.org/10.1016/j.csbj.2014.11.005

Lasi, H., Fettke, P., Kemper, H.G., Feld, T. \& Hoffmann, M. (2014). Industry 4.0. Business \& Information Systems Engineering, 6(4), 239-242. https://doi.org/10.1007/s12599014-0334-4

Lee, J., Davari, H., Singh, J. \& Pandhare, V. (2018). Industrial artificial intelligence for industry 4.0-based manufacturing systems. Manufacturing Letters, 18, 20-23.

Li, B., Hou, B., Yu, W., Lu, X. \& Yang, C. (2017). Applications of artificial intelligence in intelligent manufacturing: $\mathrm{A}$ review. 
Frontiers of Information Technology \& Electronic Engineering, 18(1), 86-96. https://doi.org/10.1631/FITEE.1601885

Lu, H., Li, Y., Chen, M., Kim, H. \& Serikawa, S. (2018). Brain Intelligence: Go beyond Artificial Intelligence. Mobile Networks and Applications, 23(2), 368-375. https://doi.org/10.1007/s11036-017-0932-8

Maimun, N.H.A., Ismail, S., Junainah, M., Razali, M.N., Tarmidi, M.Z. \& Idris, N.H. (2018). An integrated framework for affordable housing demand projection and site selection. IOP Conference Series: Earth and Environmental Science, $\quad 169$, 012094. https://doi.org/10.1088/17551315/169/1/012094

Mann, M. \& Smith, M. (2017). Automated Facial Recognition Technology: Recent Developments and Approaches to Oversight. University of New South Wales Law Journal, 40, 121.

Masuzawa, H., Miura, J. \& Oishi, S. (2017). Development of a mobile robot for harvest support in greenhouse horticulture-Person following and mapping. 2017 IEEE/SICE International Symposium on System Integration (SII), 541-546. https://doi.org/10.1109/SII.2017.8279277

Maylawati, D.S., Ramdhani, M.A., Zulfikar, W.B., Taufik, I. \& Darmalaksana, W. (2017). Expert system for predicting the early pregnancy with disorders using artificial neural network. 2017 5th International Conference on Cyber and IT Service Management (CITSM), 1-6. https://doi.org/10.1109/CITSM.2017.80892 43

Mgala, M. \& Mbogho, A. (2015). Data-driven Intervention-level Prediction Modeling for Academic Performance. Proceedings of the Seventh International Conference on Information and Communication Technologies and Development, 2:1-2:8. https://doi.org/10.1145/2737856.2738012

Morano, P., Tajani, F. \& Torre, C.M. (2015). Artificial intelligence in property valuations an application of artificial neural networks to housing appraisal. Adv. Environ. Sci. Energy Plan, 2015, 23-29.

Nabi, M.K. (2019). The Impact of Artificial Intelligence (AI) on Workforce in Emerging Economies. Global Journal of Management
And Business Research. ttps://www.journalofbusiness.org/index.p hp/GJMBR/article/view/2802

Neethirajan, S., Tuteja, S.K., Huang, S.T. \& Kelton, D. (2017). Recent advancement in biosensors technology for animal and livestock health management. Biosensors and Bioelectronics, 98, 398-407. https://doi.org/10.1016/j.bios.2017.07.015

$\mathrm{Ng}$, A. (2017). Why AI is the new electricity.

Shamshiri, R., Weltzien, C., Hameed, I.A., Yule, J.I., Grift, E.T., Balasundram, S.K., Pitonakova, L., Ahmad, D. \& Chowdhary, G. (2018). Research and development in agricultural robotics: A perspective of digital farming. 114.

https://doi.org/10.25165/j.ijabe.20181104.4 278

Stearns, P.N. (2018). The Industrial Revolution in World History. Routledge. https:// doi.org/10.4324/9780429494475

Tanaka, K. \& Sakata, I. (2016). Researcher qualitative change by governmental support in Japan. 2016 IEEE International Conference on Industrial Engineering and Engineering Management (IEEM), 1800-1803. https://doi.org/10.1109/IEEM.2016.779818 8

Wuest, T., Weimer, D., Irgens, C. \& Thoben, K.D. (2016). Machine learning in manufacturing: Advantages, challenges, and applications. Production \& Manufacturing Research, 4(1), 23-45.

https://doi.org/10.1080/21693277.2016.119 2517 